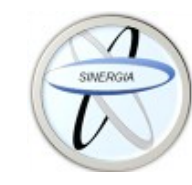

\title{
Generalidades sobre toxicidad serotoninérgica
}

\author{
Overview of serotonin toxicity
}

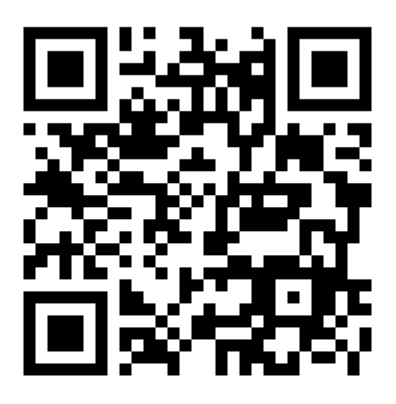

Recibido 05/08/2021

\author{
${ }^{1}$ Dr. Roberto Orozco García \\ Investigador independiente, Alajuela, Costa Rica \\ (D) https://orcid.org/0000-0003-4641-5001 \\ ${ }^{2}$ Dr. Juan Carlos Garnier Fernández \\ Investigador independiente, San José, Costa Rica \\ (D) https://orcid.org/0000-0002-5716-4562 \\ ${ }^{3}$ Dra. Gloriana Pizarro Alvarado \\ Investigadora independiente, Heredia, Costa Rica \\ (D) https://orcid.org/0000-0002-9178-2751
}

Corregido 02/09/2021
Aceptado

20/xx/2021

\section{RESUMEN}

El síndrome serotoninérgico / intoxicación serotoninérgica es un cuadro clínico potencialmente mortal, desencadenado por el uso de fármacos con actividad sobre la serotonina, o bien, sobre sus receptores, en tanto que puede ocurrir mediante el uso terapéutico de este tipo de fármacos, sobredosis intencional o mediante la interacción farmacológica a través de distintos mecanismos de acción farmacológica. El cuadro clínico se presenta como un conjunto de alteraciones a nivel del estado mental, hiperactividad neuromuscular y una profusa sobreactividad del sistema nervioso autónomo. Además, el diagnóstico se basa en historia clínica y examen físico detallados con énfasis en historial de consumo de fármacos y apoyado en pruebas de laboratorio. El tratamiento está basado, principalmente, en medidas de soporte y el antagonismo farmacológico, con vital importancia en el reconocimiento y manejo temprano para evitar las complicaciones.

PALABRAS CLAVE: serotoninérgicos; intoxicación; serotonina; diagnóstico; manejo.

\section{ABSTRACT}

Serotonin syndrome / serotonin intoxication is a life-threatening clinical condition, triggered by the use of drugs with serotonin activity or its activity on serotoninergic receptors, while it can occur through the therapeutic use of this types of drugs, it can also occur by intentional overdose or through pharmacological interaction through different pharmacological mechanisms of action. The clinical picture presents as a combination of mental status changes, 
neuromuscular hyperactivity and a profuse activity of the autonomic nervous system. Diagnosis is based on a detailed medical history and physical examination with an emphasis on ongoing medications and drug use and supported by laboratory tests. Treatment is based mainly on supportive measures and pharmacological antagonism, with vital importance in early recognition and management to avoid complications.

KEYWORDS: serotonin agents; poisoning; serotonin; diagnosis, treatment.

\footnotetext{
${ }^{1}$ Médico general, graduado de la Universidad de Iberoamérica (UNIBE). Cód. MED16746. Correo: rob.orozco.garcia@gmail.com

${ }^{2}$ Médico general, graduado de la Universidad de Iberoamérica (UNIBE). Cód. MED16727. Correo: juankgarnier@gmail.com

${ }^{3}$ Médica general, graduada de la Universidad de Iberoamérica (UNIBE). Cód. MED16722. Correo: gloripa17@gmail.com
}

\section{INTRODUCCIÓN}

El síndrome serotoninérgico (SS) es un término que hace referencia a la presentación de un grupo de signos y síntomas neuromusculares, autonómicos y de afectación del estado mental, resultado de una excesiva actividad serotoninérgica en el sistema nervioso central secundario a la administración de drogas con actividad serotoninérgica $(1,2)$.

Es conveniente señalar que expertos en el área de la toxicología han venido impulsando el uso del término toxicidad por serotonina (TS) o toxicidad serotoninérgica en un intento de abandonar el uso inexacto del término SS, para referirse a este espectro de signos y síntomas resultado del exceso de serotonina a nivel de neuronas postsinápticas (3). En cuanto a este término, TS hace alusión a que el mecanismo desencadenante es una forma de envenenamiento, y que no se preste a la malinterpretación de considerarlo como una reacción idiosincrásica a medicamentos (4). Con base en lo anteriormente explicado, para efectos de esta revisión, se continuará refiriéndose a este toxíndrome como TS.

La cantidad de medicamentos causantes de TS es extensa, esto en cuanto a que el exceso de estimulación de receptores de serotonina puede alcanzarse por distintas categorías de medicamentos, cuyas composiciones químicas pueden ser incluso completamente distintas entre ellas.

Lo anterior responde a los diversos blancos farmacológicos donde pueden actuar los fármacos con acción serotoninérgica, desde fármacos agonistas directos de receptores de serotonina, hasta los que disminuyen la descomposición del neurotransmisor. Desde sustancias de abuso, medicamentos utilizados para tratamiento de patologías médicas y psiquiátricas, e incluso suplementos dietéticos, se han reportado como implicados en el desarrollo de TS (5). La incidencia de TS es un dato inexacto, puesto que el diagnóstico, a pesar de la existencia de herramientas diagnósticas, es enteramente clínico, no existe una prueba de laboratorio; esto, aunado al subdiagnóstico de casos que se presentan con síntomas leves, síntomas muy variados o por clínicos sin el conocimiento para identificarlo, hace que no solo la incidencia verdadera sea un dato desconocido, sino que el desenlace de pacientes con TS puede llegar a tener complicaciones fatales, si no se realiza el correcto diagnóstico y se da el manejo adecuado (6).

Por este motivo, es de suma importancia, y el objetivo de la presente revisión, presentar de forma práctica la epidemiología, fisiopatología, fármacos más comúnmente implicados, características clínicas, diagnóstico, diagnóstico diferencial y manejo del TS. 


\section{MÉTODO}

Para la elaboración del presente artículo, se utilizaron bibliografías tomadas de bases de datos de Pubmed, Google Scholar, Medline (NLM), y se seleccionaron publicaciones a partir de los 5 años más recientes, basado en los términos: "serotoninergic syndrome", "serotonergic toxicity", "serotonin toxicity" y "serotonin intoxication". Además, se agregaron términos a la búsqueda para mejorar especificidad: "síntomas", "causas", "diagnóstico" y "tratamiento".

Se depuraron los resultados eliminando aquellos artículos no competentes al tema de revisión y aquellos que presentaban duplicados, en total se toman 22 artículos. A lo anterior, se adjunta una referencia externa de la búsqueda a través de los motores de búsqueda, una herramienta epidemiológica de incidencia de casos de la FDA, referencia utilizadas en la epidemiología.

\section{EPIDEMIOLOGÍA}

En los últimos años, la incidencia de TS ha tenido un importante aumento, según la FDA Adverse Events Reporting System (FAERS), en los Estados Unidos de América solamente para el 2020 hubo un incremento en la incidencia de casos de un $11.4 \%$ respecto al 2019, a su vez, el 2019 tuvo un incremento de $17.8 \%$ respecto al 2018 (7). Lo anterior obedece a distintos motivos: el primero es que la incidencia de TS discurre en paralelo con el incremento en el diagnóstico y tratamiento farmacológico del trastorno depresivo mayor en la población estadounidense; el segundo es el aumento de prescripción fuera de prospecto "Offlabel" por parte de profesionales en salud mental y atención primaria; el tercero es el incremento en el uso de sustancias de abuso; el cuarto es el aumento de intentos de autoeliminación con medicamentos antidepresivos; por último, y de suma importancia sobre todo en el ámbito intrahospitalario, el incremento en el uso de opioides en el manejo del dolor $(4,8,9)$.

\section{FISIOPATOLOGÍA}

Como se describió previamente, el mecanismo principal de TS es un exceso de serotonina, por lo tanto, es fundamental un entendimiento de la fisiología de esta.

Serotonina o 5-Hidroxitriptamina (5-HT), es un neurotransmisor tipo monoamina derivado de la hidroxilación y descarboxilación del aminoácido esencial triptófano, reacción catalizada por la enzima triptófano hidroxilasa. Esta reacción enzimática se lleva a cabo en dos regiones del cuerpo: a nivel del SNC, por medio de neuronas localizadas en los núcleos del rafé en la línea media del tallo cerebral y a nivel te tejidos periféricos, a partir de las células enterocromafines del tracto gastrointestinal (10). Posterior a la síntesis de 5-HT, esta será almacenada en vesículas de células presinápticas para su posterior liberación en la hendidura sináptica, por medio del proceso de exocitosis, y lograr su blanco efector en los receptores de serotonina de las células postsinápticas. Este proceso posee distintos mecanismos control que sirven como reguladores, evitando así una exagerada respuesta fisiológica secundaria a sobreestimulación de receptores serotoninérgicos.

El primero de estos mecanismos por mencionar ocurre al liberarse $5-\mathrm{HT}$ en la hendidura sináptica, no solamente se acopla 5-HT en los receptores postsinápticos, sino que, además, también hay acople y activación de autorreceptores presinápticos de 5-HT, los cuales inhiben la exocitosis de más 5-HT. Otro mecanismo control es el final de su acción por medio de la recaptación de 5-HT por parte de una proteína transportadora de este localizada en la membrana celular, cuya función corresponde a la de transportar 5-HT previamente liberado a la hendidura 
sináptica hacia el interior de la neurona presináptica nuevamente, donde será metabolizada a ácido 5-hidroxindolacético por la enzima intracelular monoamino oxidasa $A(11-13)$.

Actualmente, se conocen siete clases de receptores para 5-HT, se clasificaron a partir de las respuestas fisiológicas manifestadas tras su estimulación, de modo que existe una amplia gama de funciones fisiológicas que desempeña la 5-HT; de estos receptores, el que más se ha podido identificar como mayormente relevante para la aparición de la intoxicación de serotonina es el 5-HT-2a. Algunas de estas funciones a nivel del SNC son la regulación del estado anímico, ciclo circadiano, apetito, comportamiento sexual, temperatura corporal, percepción y modulación del dolor y mecanismo de emesis. Además, a nivel periférico, la 5-HT se ve implicada en la regulación de la motilidad gastrointestinal, el control vasomotor e incluso a nivel de la activación de plaquetas y hemostasia (10).
Fármacos desencadenantes de TS pueden ser muy distintos entre ellos, desde distintas clases y categorías, incluso diferentes indicaciones, pero todos estos comparten finalmente la misma acción: aumentar concentraciones de 5-HT. Lo anterior puede ser resultado de distintos mecanismos inherentes a la farmacología de cada fármaco; es posible divisar cinco mecanismos farmacológicos por medio de los cuales distintos grupos de fármacos pueden resultar en TS: aumento en la síntesis de $5-\mathrm{HT}$, inhibición del metabolismo de 5-HT, aumento de la liberación de 5-HT, agonismo/activación directa sobre receptores de 5 - $\mathrm{HT}$ y disminución en la recaptura de 5-HT (tabla 1). Por lo general, el desarrollo de TS no necesariamente ocurre por la sobredosis de un solo fármaco con efecto serotoninérgico, la mayoría de las ocasiones es por la interacción de fármacos con mecanismos distintos entre sí; así mismo, esto viene a recalcar que la naturaleza de los efectos de TS es dosis dependiente (12).

\begin{tabular}{|l|l|}
\hline $\begin{array}{l}\text { Tabla 1. Mecanismos involucrados en término de toxicidad por serotonina y los fármacos } \\
\text { asociados }\end{array}$ \\
\hline Aumento en la síntesis de 5-HT & L-triptófano, cocaína, fentermina \\
\hline Inhibición del metabolismo de 5-HT & $\begin{array}{l}\text { Buspirona, St. John's Wort, fármacos con actividad } \\
\text { inhibidora de la monoamino oxidasa (fenelzina, azul de } \\
\text { metileno, linezolid). Triptánes. }\end{array}$ \\
\hline Aumento de la liberación de 5-HT & $\begin{array}{l}\text { Meperidina, oxicodona, tramadol, MDMA, } \\
\text { dextrometorfano. }\end{array}$ \\
\hline Agonistas directos receptores 5-HT & $\begin{array}{l}\text { Metoclopramida, fentanil, meperidina, triptánes, } \\
\text { dihidroergotamina, litio. }\end{array}$ \\
\hline Disminución en la recaptura de 5-HT & $\begin{array}{l}\text { Inhibidores selectivos de recaptura de serotonina } \\
\text { (citalopram, escitalopram, fluoxetina, paroxetina), } \\
\text { inhibidores de la recaptura de serotonina-norepinefrina } \\
\text { (duloxetina, venlafaxina), antidepresivos tricíclicos } \\
\text { imipramina, clomipramina), MDMA, } \\
\text { (amitriptilina, } \\
\text { granisetrón, ondansetrón. }\end{array}$
\end{tabular}

Fuente. Adaptado de Francescangeli J, Karamchandani K, Powell M, Bonavia A. The Serotonin Syndrome: From Molecular Mechanisms to Clinical Practice. Int J Mol Sci. 2019 May 9;20(9):228 


\section{MANIFESTACIONES CLÍNICAS}

Como se mencionó anteriormente, el diagnóstico de TS es completamente clínico, basado en una historia clínica detallada, con énfasis en uso de medicamentos o sustancias de abuso, así como una examinación física y neurológica adecuada.

Se ha hecho énfasis, en distintas revisiones y en textos de formación médica, sobre que los síntomas de TS suelen ocurrir a las 24 horas de la administración del agente desencadenante $(1,5,12-15)$, a tal punto que este intervalo se toma en cuenta dentro de las consideraciones para el diagnóstico. Según Scotton et al., esta noción se origina de una revisión de 41 casos de TS entre 1995 a 1999 (11). En un metaanálisis más reciente de casos, Werneke et al. identificaron que, en más del $60 \%$ de los casos, de un total de 299, se presentaron síntomas a las 6 horas de la administración del fármaco (16).

Comúnmente, las características clínicas de TS se han agrupado en una triada: alteraciones del estado mental, inestabilidad autonómica, e hiperactividad neuromuscular (15). Más concretamente, los datos clínicos que se han reportan asociados a alteraciones del estado mental son: insomnio, ansiedad, agitación, confusión, letargo; los signos y síntomas de inestabilidad autonómica incluyen: diaforesis, midriasis, taquicardia, hipertermia, hipertensión, vómitos hiperactividad del tracto gastrointestinal e incluso diarrea; $y$ los referentes a hiperactividad neuromuscular son: rigidez muscular, akathisia, clonus, convulsiones, hiperreflexia e incluso un signo Babinski bilateral $(2,17,18)$. Por su parte, Prakash et al. realizaron una revisión sistemática de 56 casos de TS reportados en la literatura que concluyeron en fatalidad, se adjunta una tabla con las características clínicas más comunes de estos casos (tabla 2) (2).

La presentación es altamente variable, abarcando desde sintomatología leve hasta severa, e incluso poniendo en riesgo la vida del paciente; conforme aumenta la severidad del cuadro es cuando pueden empezar a surgir las complicaciones severas, entre ellas: acidosis metabólica, rabdomiólisis, falla renal, síndrome de distrés respiratorio agudo y muerte. Lo anterior es reflejo del nivel de sobreactivación serotoninérgica que está ocurriendo, razón por la cual se dificulta en muchas ocasiones realizar el preciso diagnóstico al momento de la valoración inicial, en tanto que pueden presentarse con cuadros clínicos subagudos que pueden distraer y confundirse con otras entidades (14).

\begin{tabular}{|c|c|c|c|c|c|}
\hline Hipertermia & $61 \%$ & - Midriasis & $25 \%$ & - Diarrea & $9 \%$ \\
\hline - Convulsiones & $36 \%$ & - Hipotensión & $20 \%$ & - Clonus ocular & $7 \%$ \\
\hline - Taquicardia & $36 \%$ & - Mioclonía & $20 \%$ & - Hipertensión & $7 \%$ \\
\hline - Tremor & $30 \%$ & - Agitación & $18 \%$ & - Cefalea & $5 \%$ \\
\hline - Diaforesis & $29 \%$ & - Clonus & $14 \%$ & - Escalofríos & $5 \%$ \\
\hline - $\quad$ Rigidez & $27 \%$ & - Confusión & $13 \%$ & - In-coordinación & $5 \%$ \\
\hline - Hiperreflexia & $27 \%$ & - Trismus & $11 \%$ & - Opistótonos & $0 \%$ \\
\hline
\end{tabular}

Fuente. Adaptado de Prakash S, Rathore C, Rana K, Prakash A. Fatal serotonin syndrome: a systematic review of 56 cases in the literature. Clin Toxicol. 2021 Feb 1;59(2):89-100. 


\section{DIAGNÓSTICO}

A raíz del reto diagnóstico que representa la TS, por motivos previamente discutidos, se han desarrollado varias herramientas diagnósticas basadas en el hallazgo 0 cumplimiento de criterios clínicos, entre ellas existen los criterios de Sternbach, Radomski y el Hunter Serotonin Toxicity Criteria o (HSTC). Este último fue recientemente desarrollado tras estudiar los hallazgos clínicos de 2222 casos de sobredosis por drogas con actividad serotoninérgica $y$ deriva en un modelo de siete características clínicas y un sistema de descarte "RuleIn/Rule Out". Al utilizarse, ha demostrado poseer un alto índice predictivo para TS con una sensibilidad de $84 \%$ y especificidad de $97 \%$ (figura 1) $(13,19,20)$.

No existe una prueba de laboratorio confirmatoria y el diagnóstico se hace de forma clínica. Parte del abordaje inicial para evaluar al paciente amerita de un hemoleucograma, electrolitos, pruebas de función renal, creatinin fosfokinasa, pruebas de función hepática, pruebas de coagulación, urianálisis, un análisis de tóxicos y una punción lumbar. Además, pacientes pueden presentar alteraciones en pruebas de laboratorio y dichas alteraciones son reflejo de la severidad asociada a disfunción orgánica, entre ellas: acidosis metabólica, aumento de transaminasas, aumento en la creatinina y leucocitosis (15).

Figura 1. Diagnóstico de toxicidad por serotonina

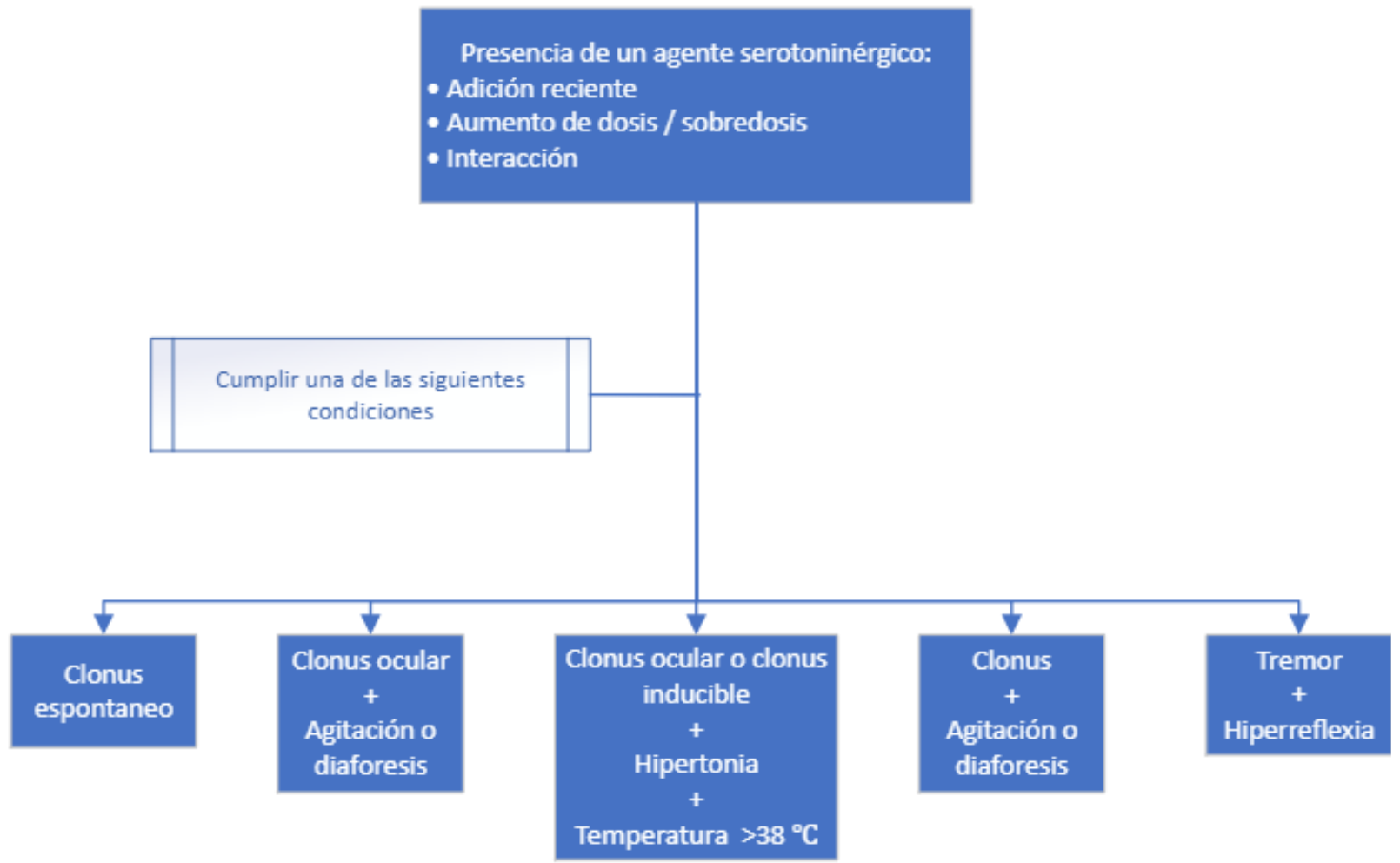

Fuente. Adaptado de Bartakke A, Corredor C, van Rensburg A. Serotonin syndrome in the perioperative period. BJA Educ. 2020 Jan;20(1):10-7 


\section{DIAGNÓSTICO DIFERENCIAL}

Existen varias enfermedades que comparten signos y síntomas similares a la TS, las cuales deben siempre incluirse en el diferencial por su potencialmente fatal desenlace, si no se identifican de forma precisa, consisten en: síndrome neuroléptico maligno (SNM), toxicidad anticolinérgica, hipertermia maligna, meningitis y encefalitis (tabla 3) (11).

SNM es una reacción idiosincrásica que pone en riesgo la vida del paciente secundario a dosis terapéuticas de antagonistas de dopamina, más comúnmente antipsicóticos típicos (haloperidol, flufenazina), pero también puede ocurrir con antipsicóticos atípicos (clozapina, risperidona, olanzapina). Se desconoce el mecanismo fisiopatológico exacto del SNM, pero el bloqueo de la vía dopaminérgica es clave en su génesis. La historia clínica del paciente, tiempo de inicio de síntomas, historial medicamentoso y hallazgos en la examinación clínica permiten diferenciar estas dos condiciones.

El SNM es de inicio lento, días a semanas, los medicamentos son diferentes y, mientras que TS se caracteriza por hiperreactividad neuromuscular (tremor, clonus, hiperreflexia, nistagmus), en SNM hay hipoactividad neuromuscular con hallazgos extrapiramidales (hipoquinesia y rigidez); no hay hiperreflexia ni clonus $(5,19,21)$.

En la toxicidad anticolinérgica, las diferencias que permiten distinguirlo de una TS son una actividad neuromuscular sin alteración, peristalsis disminuida, mucosas y piel secas con hiperemia. Los otros signos y síntomas pueden compartirse entre ambas entidades (5).

Por su parte, la hipertermia maligna es una miopatía subclínica en la cual fármacos de tipo anestésicos generales halogenados y relajantes musculares de tipo despolarizantes desencadenan una contracción excesiva del músculo esquelético que lleva a un estado hipercatabólico y aumento de temperatura corporal que ponen en riesgo la vida. Es de inicio súbito tras la exposición a los agentes previamente mencionados. Se diferencia en que hay hiporreflexia, aumento en las concentraciones del $\mathrm{CO}_{2}$ al final de una respiración tidal y hay presencia de livedo reticularis a nivel de piel (11).

\section{MANEJO}

Actualmente, distintas organizaciones y expertos en toxicología clínica abogan por un modelo basado en prevención y vigilancia farmacológica para pacientes en tratamiento con fármacos de alto riesgo para TS. Estas estrategias involucran la evaluación de la necesidad de permanecer utilizando estos medicamentos; el uso de las dosis más bajas requeridas para el manejo adecuado de los síntomas de la patología por la cual se está usando el medicamento; un enfoque a la historia médica de medicamentos a los cuales el paciente está sujeto $\mathrm{y}$, así mismo, al uso de sustancias ilícitas, citas control a corto plazo durante cambios en la dosificación; vigilancia médica estricta en pacientes hospitalizados expuestos a medicamentos desencadenantes $\mathrm{y}$, sobre todo, educación al paciente dirigida al reconocimiento temprano $(12,14)$.

Por otra parte, de los pacientes que se presentan con TS instaurada, el 70\% resolverá su cuadro en las $24 \mathrm{~h}$ siguientes de hipertermia secundaria a la contracción muscular sostenida por la hipertonía $(6,12)$.

Dicho lo anterior, las benzodiacepinas favorecen la disminución de la presión arterial y frecuencia cardíaca. Uno de los medicamentos que actualmente ha ganado atención es el agonista adrenérgico de receptores alfa tipo 2 , dexmedetomidina, utilizado en unidades de cuidados intensivos y anestesia. Su perfil farmacológico lo hace favorable para el tratamiento de TS. 


\begin{tabular}{|c|c|c|c|c|c|c|}
\hline Toxíndrome & $\begin{array}{l}\text { Agente } \\
\text { causal }\end{array}$ & Inicio & $\begin{array}{l}\text { Signos } \\
\text { vitales }\end{array}$ & $\begin{array}{l}\text { Pupila } \\
\text { s }\end{array}$ & $\begin{array}{l}\text { Estado } \\
\text { mental }\end{array}$ & Clínica \\
\hline $\begin{array}{l}\text { Toxicidad } \\
\text { serotoninérgic } \\
\text { a }\end{array}$ & $\begin{array}{l}\text { Drogas } \\
\text { serotoninérgica } \\
\mathrm{s}\end{array}$ & $\begin{array}{l}\text { Súbito } \\
<24 \mathrm{~h}, \\
\text { mayoría } \\
\text { resuelve } \\
\text { en } 24 \mathrm{~h} \\
\text { con } \\
\text { tratamient } \\
\text { o }\end{array}$ & $\begin{array}{l}\text { Hipertermia } \\
>41.1^{\circ} \mathrm{C}, \\
\text { taquicardia, } \\
\text { hipertensión } \\
\text {, taquipnea }\end{array}$ & $\begin{array}{l}\text { Midriasi } \\
\mathrm{s}\end{array}$ & $\begin{array}{l}\text { Delirium, } \\
\text { agitación y } \\
\text { coma }\end{array}$ & $\begin{array}{l}\text { Hiperreactivida } \\
\mathrm{d} \\
\text { neuromuscular, } \\
\text { inestabilidad } \\
\text { autonómica, } \\
\text { peristaltismo } \\
\text { aumentado }\end{array}$ \\
\hline $\begin{array}{l}\text { Síndrome } \\
\text { neuroléptico } \\
\text { maligno }\end{array}$ & $\begin{array}{l}\text { Antagonistas } \\
\text { dopaminérgicos } \\
\text { y suspensión } \\
\text { de drogas } \\
\text { dopaminérgicas }\end{array}$ & $\begin{array}{l}\text { Inicio lento } \\
\text { (días a } \\
\text { semanas) } \\
\text { hasta } 10 \\
\text { días para } \\
\text { resolver } \\
\text { con } \\
\text { tratamient } \\
\text { o }\end{array}$ & $\begin{array}{l}\text { Hipertermia } \\
>41.1^{\circ} \mathrm{C}, \\
\text { taquicardia, } \\
\text { hipertensión } \\
\text { y taquipnea }\end{array}$ & $\begin{array}{l}\text { Normal / } \\
\text { midriasi } \\
\text { s }\end{array}$ & $\begin{array}{l}\text { Delirium, } \\
\text { agitación }\end{array}$ & $\begin{array}{l}\text { Hipoactividad } \\
\text { neuromuscular } \\
\text { (bradiquinesia, } \\
\text { rigidez } \\
\text { muscular), } \\
\text { peristaltismo } \\
\text { disminuido }\end{array}$ \\
\hline $\begin{array}{l}\text { Toxicidad } \\
\text { anticolinérgica }\end{array}$ & $\begin{array}{l}\text { Agentes } \\
\text { anticolinérgicos }\end{array}$ & $\begin{array}{l}\text { Súbito } \\
<24 \mathrm{~h}, \\
\text { resuelve } \\
\text { en horas a } \\
\text { días con } \\
\text { tratamient } \\
\text { o }\end{array}$ & $\begin{array}{l}\text { Hipertermia, } \\
\text { taquicardia, } \\
\text { hipertensión } \\
\text { leve y } \\
\text { taquipnea }\end{array}$ & $\begin{array}{l}\text { Midriasi } \\
\mathrm{s}\end{array}$ & $\begin{array}{l}\text { Hiperventilación } \\
\text {, agitación, } \\
\text { delirium, } \\
\text { alucinaciones }\end{array}$ & $\begin{array}{l}\text { Neuromuscular } \\
\text { normal, } \\
\text { mucosas y piel } \\
\text { seca con } \\
\text { hiperemia, } \\
\text { peristalsis } \\
\text { disminuida, } \\
\text { mioclonía, } \\
\text { retención } \\
\text { urinaria }\end{array}$ \\
\hline $\begin{array}{l}\text { Hipertermia } \\
\text { maligna }\end{array}$ & $\begin{array}{l}\text { Anestésicos } \\
\text { inhalados y } \\
\text { relajantes } \\
\text { musculares de } \\
\text { tipo } \\
\text { despolarizantes }\end{array}$ & $\begin{array}{l}\text { Súbito, } \\
\text { resuelve } \\
\text { en 24-48h } \\
\text { con } \\
\text { tratamient } \\
\text { o }\end{array}$ & $\begin{array}{l}\text { Hipertermia, } \\
\text { taquicardia, } \\
\text { hipertensió, } \\
\text { taquipnea }\end{array}$ & Normal & Agitación & $\begin{array}{l}\text { Rigidez } \\
\text { muscular } \\
\text { generalizada, } \\
\text { contracción de } \\
\text { músculos } \\
\text { maseteros, } \\
\text { livedo } \\
\text { reticularis, } \\
\text { hiporreflexia. }\end{array}$ \\
\hline
\end{tabular}

Fuente. Adaptado de Prakash S, Rathore C, Rana K, Prakash A. Fatal serotonin syndrome: a systematic review of 56 cases in the literature. Clin Toxicol. 2021 Feb 1;59(2):89-100

Al ser un agonista de receptor alfa tipo 2, inhibe la liberación de serotonina por parte de neuronas serotoninérgicas y tiene el beneficio agregado de producir sedación, ansiólisis y disminución de la taquicardia e hipertensión (13).

haber suspendido el medicamento desencadenante, variando hasta 3 días de acuerdo con la vida media y los metabolitos activos de la droga en específico; el $40 \%$ requerirá una unidad de cuidados intensivos y un $25 \%$ de pacientes requerirá intubación endotraqueal (12).
Encima de suspender el agente desencadenante, el manejo del paciente con TS gira en torno a tratamiento de soporte, corrección de signos vitales, fluido terapia, manejo de la agitación, hipertermia y la inestabilidad autonómica.

Uno de los pilares en el manejo es el tratamiento de la agitación y ansiedad por medio del uso de fármacos del tipo benzodiacepinas (lorazepam, midazolam y diazepam). En la actualidad, la literatura científica no fomenta el uso de una benzodiacepina sobre otra, aunque en 
estudios animales, el uso de lorazepam desfavorece la aparición de hipertermia. Sedar al paciente favorece la disminución del riesgo a hacer acidosis láctica severa e hipertermia secundaria a la contracción muscular sostenida por la hipertonía $(6,12)$. Dicho lo anterior, las benzodiacepinas favorecen la disminución de la presión arterial y frecuencia cardíaca. Uno de los medicamentos que actualmente ha ganado atención es el agonista adrenérgico de receptores alfa tipo 2, dexmedetomidina, utilizado en unidades de cuidados intensivos y anestesia. Su perfil farmacológico lo hace favorable para el tratamiento de TS. Al ser un agonista de receptor alfa tipo 2, inhibe la liberación de serotonina por parte de neuronas serotoninérgicas $y$ tiene el beneficio agregado de producir sedación, ansiólisis y disminución de la taquicardia e hipertensión (13).

Sobre la inestabilidad autonómica en pacientes con TS severo, en los cuales el uso de benzodiacepinas no logró estabilizarlo, la literatura recomienda el uso de agentes de acción corta como esmolol o nitroprusiato de sodio. En caso de cursar con hipotensión, el tratamiento de preferencia son dosis bajas de simpaticomiméticos de acción directa como norepinefrina, fenilefrina o epinefrina. Además, se debe evitar el uso de agentes simpaticomiméticos indirectos como la dopamina, sobre todo cuando está involucrada la presencia de inhibidores de la enzima monoamino oxidasa a razón de una respuesta hemodinámica excesiva (11).

La hipertermia es, en principio, mediada por la hiperactividad del músculo esquelético, razón por la cual, en casos severos de TS, puede llevar al aumento de la enzima creatinin quinasa reflejando un proceso de rabdomiólisis, por consiguiente, es clave, en el manejo y la prevención de falla orgánica multisistémica, la disminución de la hipertermia.

Como se mencionó, la razón de la hipertermia no es secundaria a liberación de citoquinas o mediadores inflamatorios que afecten el termostato hipotalámico, es secundaria a contracción muscular isovolumétrica sostenida, de modo que antipiréticos como el acetaminofén no van a tener efecto. Elementos citados en la literatura que han demostrado mejorar la sobrevida mediante la reducción de la temperatura han sido la sedación, el enfriamiento activo mediante uso de cualquier método que se tenga a disposición, y en casos extremos, la parálisis muscular y ventilación mecánica asistida en una unidad de cuidados intensivos. En este último escenario, la literatura hace la observación respecto a ciertos medicamentos por utilizar en la parálisis neuromuscular y sedo-analgesia durante la intubación y ventilación mecánica. No se debe utilizar relajantes musculares del tipo despolarizantes por dos razones de importancia:

1. Corre el riesgo de empeorar rabdomiólisis

2. Estos medicamentos tienen el riesgo de producir hiperkalemias, además, en situaciones de rabdomiólisis, pueden llevar a arritmias cardíacas fatales.

También recordar que hay opioides, sobre todo del grupo de las piperidinas como el fentanilo, que tienen efecto serotoninérgico, por lo cual deben ser evitados $(2,5,11,13,20,22)$.

El fármaco que se considera antídoto en la TS es la ciproheptadina. Esta es un antagonista del receptor $\mathrm{H} 1$ que ha demostrado tener propiedades antagonistas no selectivas para receptores de 5-HT, sobre todo para el receptor 5-HT-2A y ha sido útil en el alivio de síntomas de TS. La dosis que se utiliza es de $12 \mathrm{mg}$ seguida por $2 \mathrm{mg}$ cada 2 horas hasta que haya mejoría clínica. No obstante, la ciproheptadina tiene la limitante de existir solo en presentación oral, pero puede triturarse y administrarse vía sonda nasogástrica (13). 


\section{CONCLUSIONES}

La TS es una condición que, lejos de desaparecer o disminuir en incidencia, tomará más importancia en los años venideros, no solamente por el foco público que se ha generado alrededor de este tema en el ámbito de la toxicología, sino también por el creciente aumento en pacientes con padecimiento de trastorno depresivo mayor. El uso de medicamentos con efectos serotoninérgicos por sí solos es poco probable que ocasione TS, sobre todo cuando la mayoría de medicamentos utilizados en la población son inhibidores selectivos de la recaptura de serotonina, que ya de por sí son mucho más seguros que medicamentos de otras categorías, sin embargo, en una población que tiende a la polifarmacia y uso de medicamentos que no requieren de prescripción o vigilancia médica, el riesgo de interacciones medicamentosas que propicien el desarrollo de TS va a incrementar paralelamente. Por estas razones, es imperativo que el cuerpo de profesionales dedicados a la salud tenga presente las interacciones farmacológicas al utilizar estos medicamentos, en un afán por prevenir el desarrollo de síntomas asociados a la TS, y tener en cuenta los signos y síntomas para lograr un adecuado diagnóstico y óptimo manejo.

\section{Los autores declaran no tener conflicto de interés.}

\section{REFERENCIAS}

1. Baldo BA, Rose MA. The anaesthetist, opioid analgesic drugs, and serotonin toxicity: a mechanistic and clinical review. $\mathrm{Br} \mathrm{J}$ Anaesth. 2020 Jan;124(1):44-62.

2. Prakash S, Rathore C, Rana K, Prakash A. Fatal serotonin syndrome: a systematic review of 56 cases in the literature. Clin Toxicol. $2021 \mathrm{Feb}$ 1;59(2):89-100.

3. Macedo D, Filho AJMC, Soares de Sousa CN, Quevedo J, Barichello T, Júnior HVN, et al. Antidepressants, antimicrobials or both? Gut microbiota dysbiosis in depression and possible implications of the antimicrobial effects of antidepressant drugs for antidepressant effectiveness. J Affect Disord. 2017 Jan;208:2232.

4. Talton C. Serotonin Syndrome/Serotonin Toxicity. Fed Pract [Internet]. 2020 Oct 10 [cited 2021 Mar 20];(Vol 37 No 10). Available from: https://www.mdedge.com/fedprac/article/229630/ hospital-medicine/serotonin-syndrome/serotonintoxicity

5. Bartlett D. Drug-Induced Serotonin Syndrome. Crit Care Nurse. 2017 Feb 1;37(1):49-54.

6. Wang RZ, Vashistha V, Kaur S, Houchens NW. Serotonin syndrome: Preventing, recognizing, and treating it. Cleve Clin J Med. 2016 Nov;83(11):810-7.

7. FDA Adverse Events Reporting System (FAERS) Public Dashboard - FDA Adverse Events Reporting System (FAERS) Public Dashboard I Sheets - Qlik Sense [Internet]. [cited 2021 Mar 21]. Available from: https://fis.fda.gov/sense/app/d10be6bb-494e4cd2-82e4-0135608ddc13/sheet/45beeb7430ab-46be-8267-5756582633b4/state/analysis

8. Hassamal S, Miotto K, Dale W, Danovitch I. Tramadol: Understanding the Risk of Serotonin Syndrome and Seizures. Am J Med. 2018 Nov;131(11):1382.e1-1382.e6.

9. Karkow DC, Kauer JF, Ernst EJ. Incidence of Serotonin Syndrome With Combined Use of Linezolid and Serotonin Reuptake Inhibitors Compared With Linezolid Monotherapy. J Clin Psychopharmacol. 2017 Oct;37(5):518-23.

10. Fouquet $G$, Coman $T$, Hermine $O$, Côté $F$. Serotonin, hematopoiesis and stem cells. Pharmacol Res. 2019 Feb;140:67-74.

11. Scotton WJ, Hill LJ, Williams AC, Barnes NM. Serotonin Syndrome: Pathophysiology, Clinical Features, Management, and Potential Future Directions. Int J Tryptophan Res. 2019 Jan;12:117864691987392.

12. Francescangeli J, Karamchandani K, Powell M, Bonavia A. The Serotonin Syndrome: From Molecular Mechanisms to Clinical Practice. Int J Mol Sci. 2019 May 9;20(9):2288.

13. Bartakke A, Corredor C, van Rensburg A. Serotonin syndrome in the perioperative period. BJA Educ. 2020 Jan;20(1):10-7.

14. Foong A-L, Grindrod KA, Patel T, Kellar J. Demystifying serotonin syndrome (or serotonin toxicity). :8.

15. Simon LV, Keenaghan M. Serotonin Syndrome. In: StatPearls [Internet]. Treasure Island (FL): StatPearls Publishing; 2021 [cited 2021 Mar 23]. Available from: http://www.ncbi.nlm.nih.gov/books/NBK482377/

16. Werneke $U$, Jamshidi F, Taylor DM, Ott $M$. Conundrums in neurology: diagnosing serotonin 
syndrome - a meta-analysis of cases. BMC Neurol. 2016 Dec;16(1):97.

17. Jurek L, Nourredine M, Megarbane B, d'Amato T, Dorey J-M, Rolland B. Le syndrome sérotoninergique: une revue actualisée de la littérature. Rev Médecine Interne. 2019 Feb;40(2):98-104.

18. Ansari H, Kouti L. Drug Interaction and Serotonin Toxicity with Opioid Use: Another Reason to Avoid Opioids in Headache and Migraine Treatment. Curr Pain Headache Rep. 2016 Aug;20(8):50.

19. Harada T, Hirosawa T, Morinaga K, Shimizu T. Metoclopramide-induced Serotonin Syndrome. Intern Med. 2017 Mar 15;56(6):737-9.

20. Uddin MF, Alweis $R$, Shah SR, Lateef $N$, Shahnawaz W, Ochani RK, et al. Controversies in Serotonin Syndrome Diagnosis and Management: A Review. J Clin Diagn Res JCDR. 2017 Sep;11(9):OE05-7.

21. Tormoehlen LM, Rusyniak DE. Neuroleptic malignant syndrome and serotonin syndrome. In: Handbook of Clinical Neurology [Internet]. Elsevier; 2018 [cited 2021 Mar 23]. p. 663-75. Available

from: https://linkinghub.elsevier.com/retrieve/pii/B9780 444640741000392

22. Bruggeman C, O'Day CS. Selective Serotonin Reuptake Inhibitor Toxicity. In: StatPearls [Internet]. Treasure Island (FL): StatPearls Publishing; 2021 [cited 2021 Mar 20]. Available from:

http://www.ncbi.nlm.nih.gov/books/NBK534815 